\title{
Discursos, reclamos y tensiones: una lectura sobre educación y diferencia ${ }^{1}$
}

\author{
David Andrés Jiménez²
}

Fecha de recepción: 26 de abril de 2015

Fecha de revisión: 27 de abril de 2015

Fecha de aprobación: 19 de mayo de 2015

\section{Resumen}

Este artículo reflexiona, a partir de cuatro investigaciones, sobre las problemáticas impuestas a la educación formal de Colombia por medio de demandas reivindicativas desde movimientos sociales que se ubican desde la diferencia. Para ello, analiza algunos discursos surgidos en las investigaciones que interpelan las instituciones educativas públicas y las tensiones que emergen en la participación de los sujetos de estos movimientos sociales en las instituciones educativas, los cuales demandan prácticas pedagógicas específicas y configuran la educación como un campo de pugna por la instauración de discursos en pos de la constitución de intersubjetividades que los defiendan y reconozcan en el ámbito social. Estos fenómenos se dan en medio de políticas insertas en la ambivalencia producida por la apuesta de una democracia que depende de paradigmas neoliberales, ante los cuales habría que hacer una discusión sobre las demandas a nuestros principios éticos y morales.

1 Artículo de reflexión que presenta resultados y posibilidades de análisis con base en cuatro investigaciones terminadas desde una perspectiva interpretativa y crítica sobre la temática. Financiación en orden investigativa: 1) Expedición Pedagógica Nacional, Universidad Nacional de Colombia; 2) Universidad Pedagógica Nacional; 3) Secretaría de Educación del Chocó-Unicef; y 4) Secretaría de Educación Distrital-IED, SaludCoop.

2 Candidato a doctor en Estudios Sociales de América Latina de la Universidad Nacional de Córdoba-Argentina, magister en Desarrollo Educativo y Social de la Universidad Pedagógica Nacional y antropólogo de la Universidad Nacional de Colombia. Docente de tiempo completo en la Maestría de Educación de la Universidad Santo Tomás, y catedrático en el programa de Trabajo Social en la Universidad de la Salle. Correo electrónico: davidjimenez@ustadistancia.edu.co; dirección de correspondencia: carrera 9A No. 63-28, Bogotá-Colombia. 


\title{
Palabras clave:
}

Desarrollo, diferencia, pobreza, cultura, pedagogía, territorio, urbano-rural, educación formal.

\section{Discourses, claims and tensions: a reading on education and difference}

\begin{abstract}
This article use the results from four investigations that the author was involved to think on the problems imposed on the formal education of Colombia by the social movements and their protests. The claims use the difference to produce demands of change. The text analyzes some speeches emerged in the researches in some public institutions. Also it reviews the tensions that emerge in the participation of people in these social movements that ask for recognition in the educational institutions. These subjects require specific teaching practices and configure the public education as a field of dispute for establishment discourses. These speeches want to build up intersubjectivities that defend them and recognize them in the social field. These phenomena occur in the middle of ambivalence policies produced by neoliberal paradigms defend one class of democracy. We should make a discussion about these demands because are related with our ethical and moral principles.
\end{abstract}

\section{Keywords:}

Development, difference, poverty, culture, education, land, urban-rural, formal education. 


\section{Discursos, reclamações e tensões: uma leitura sobre educação e diferença.}

\section{Resumo}

Este artigo reflete, a partir de quatro investigações sobre as problemáticas impostos à educação formal da Colômbia por meio das demandas de movimentos sociais que estão localizados desde a diferença. Ele analisa alguns discursos que tem surgido nas investigações que desafiam as instituições de ensino públicas e as tensões que surgem na participação dos sujeitos desses movimentos sociais em instituições educativas que exigem práticas pedagógicas específicas e configuram a educação como um campo de luta para o estabelecimento de discursos após da constituição de intersubjetividades para defendê-los e reconhecer-los no campo social. Estes fenômenos ocorrem no meio de políticas inserisse na ambivalência produzida pelo compromisso de uma democracia que depende de paradigmas neoliberais, a os quais deve fazer-se uma discussão sobre as demandas de nossos princípios éticos e morais.

\section{Palavras-chave:}

Desenvolvimento, diferença, pobreza, cultura, educação, terra, urbano-rural, educação formal.

\section{Introducción}

La educación colombiana está y estará interpelada por los reclamos desde la diferencia étnica; aspecto que demanda pensar estrategias para atender necesidades de la realidad pluriétnica, multicultural e intercultural de nuestro país. En las ciencias sociales este tipo de búsquedas han sido apoyadas por múltiples teóricos que se preguntan por "los desafíos que implica la realidad intercultural en que vivimos para repensar posibles horizontes de imaginación social y política" (Grimson, 2011 , p. 15). Afrontar estos retos pasa por comprender las dinámicas históricas que han configurado la realidad nacional y cómo las comunidades denostadas por siglos hoy buscan nuevos espacios de reconocimiento y enunciación. Por lo 
anterior, estudiar el encuentro cultural demanda reflexiones en los intereses de los sujetos, teniendo en cuenta desde dónde y cómo las realizan; reconociendo que las mismas interceptan la educación pública colombiana por ser el sistema educativo el medio más expedito para cambiar o ir cambiando las condiciones sociales adversas que enfrentan algunos grupos poblacionales. Es importante aclarar de manera enfática que el artículo únicamente aborda casos de la educación pública colombiana, la misma es entendida como "educación formal" la cual difiere de la no formal y de la informal. Carlos Calvo Muñoz en su texto Complejidad, caos y educación (2005), aclara que la educación formal y no-formal se da en ambientes escolarizados y comparten la característica de estar basados en fundamentos cartesianos, por lo que planifican las actividades en pro de garantizar el aprendizaje. Aunque, sin duda lo abordado aquí pueda tener enlaces con la educación informal o no formal estas educaciones demandan análisis propios que no son el objeto de análisis en este escrito.

Estos infortunios han generado que estas poblaciones se expresen en movimientos sociales. Este texto es parte de una reflexión más amplia sobre el ámbito educativo y el papel que tiene el sujeto en el mismo. No busca claridades definitivas, intenta plantear discusiones y organizar reflexiones en torno a la realidad de la educación formal. Pensar sobre la diferencia implica una reflexión continua y consistente sobre los sujetos producidos por las instituciones educativas. Estos sujetos se manifiestan mediante discursos que para nuestro saber pedagógico sería más que vital mapear y comprender.

En la primera parte se expondrán algunos hallazgos, cuatro investigaciones que, como se explicará luego, se consideran pertinentes para esbozar la realidad educativa del ámbito nacional, y que permitirán plantear que la educación es un campo de disputa en donde las demandas de los movimientos sociales hacen presencia. Posteriormente se identifican hallazgos significativos en estas investigaciones que permiten configurar la realidad educativa como un campo de disputa de las demandas generadas por identificaciones culturales. Los grupos de identificación cultural son muy amplios, como lo afirma Gutmann (2008):

Sus demandas los hace una categoría importante para ser considerada en la política democrática (...) [de manera general podemos ver reclamos como:] derechos de autodeterminación para el grupo, ser exceptuados de ciertas normas que generan desventajas desproporcionadas para los integrantes del grupo, ayuda financiera para las instituciones culturales del grupo y apoyo para la supervivencia cultural del grupo. No es suficiente el simple aplauso o la condena a los grupos 
de identidad cultural; antes bien, es necesario evaluar cada clase de reclamos para comprender cómo los grupos podrían propiciar y también trabar la justicia democrática (p. 63).

Sus demandas los hace una categoría importante para ser considerada en la política democrática (...) [de manera general podemos ver reclamos como:] derechos de autodeterminación para el grupo, ser exceptuados de ciertas normas que generan desventajas desproporcionadas para los integrantes del grupo, ayuda financiera para las instituciones culturales del grupo y apoyo para la supervivencia cultural del grupo. No es suficiente el simple aplauso o la condena a los grupos de identidad cultural; antes bien, es necesario evaluar cada clase de reclamos para comprender cómo los grupos podrían propiciar y también trabar la justicia democrática (p. 63).

\section{Sobre las investigaciones fuente}

Las cuatro investigaciones en las que el autor participó fueron realizadas en diferentes contextos de la educación formal colombiana. La primera en el marco de la Ruta Afrocolombiana, como parte integral de la Expedición Pedagógica Nacional (EPN) entre el 2003 y el 2004, concebida como un movimiento pedagógico colombiano, que desde 1990 hasta el 2005 acogió una masa crítica de maestros, investigadores, intelectuales y actores del sistema educativo que reflexionaron sobre el devenir de la escuela y el lugar del maestro en los múltiples modos de hacer escuela, ser maestro y construir comunidad educativa. Se buscó evidenciar las dinámicas sociales de la comunidad afro en colegios de Bogotá mediante un ejercicio de corte etnográfico, en el cual fueron observados cinco colegios de tres diferentes localidades de la ciudad (las localidades son una clasificación geográfica interna de la ciudad; Bogotá, D. C., tiene 20 localidades las cuales hacen parten de una división política, administrativa y territorial).

La investigación recolectó información sobre las dinámicas y las exigencias de la población afrocolombiana a la escuela distrital. Se utiliza el término "afrocolombiano(s)" por estar ideológicamente de acuerdo con las luchas políticas que los movimientos sociales han realizado; aunque hay sujetos que prefieren uti- 
lizar el término "negro" o quitar cualquier tipo de referencia grupal. Otra claridad escritural lleva al autor a expresar su apoyo a la lucha por la igualdad de género. Empero, para hacer la escritura amable se usará la diferencia de género cuando el caso lo amerite, de lo contrario el plural masculino referirá tanto a hombres como a mujeres indistintamente.

Los datos recabados permitieron ir más allá de los objetivos planteados inicialmente, y fue posible comprender los ejercicios de visibilización como parte de una dinámica de reconocimiento político que defendía el status de los docentes afrocolombianos y la importancia de una educación que reconociera su diferencia. Lo anterior permitió comprender que los intereses de los sujetos jugaban un papel importante en el reconocimiento político y académico; por lo cual la producción de saberes sobre la escuela y lo que en la misma hace presencia siempre sería un objetivo de estos sujetos.

Del mismo modo, las voces de la diferencia visibilizaron fenómenos como el racismo en las instituciones y permitió privilegiar ciertas voces excluidas que tenían requerimientos tanto al ámbito educativo general como a los actores que recibían o procesaban esa educación. Esta tensión enriqueció la visión de la escuela como un escenario de disputa, haciendo visibles dinámicas sociales de los "afrocolombianos al poner en juego su bagaje cultural en los planteles" (Jiménez, 2006, p. 66).

La segunda investigación tiene como marco la Línea de Educación en Valores y Convivencia Escolar de la Licenciatura en Educación Básica con Énfasis Ciencias Sociales de la Universidad Pedagógica Nacional. Sistematizar el trabajo realizado en las tesis de pregrado permitió ver tanto los requerimientos de cada investigación como de algunos grupos sociales que desde su diferencia cultural seguían cuestionando la legitimidad de la institución escolar en la formación axiológica. Cobró allí el sentido del papel de la educación en valores para la convivencia y la ciudadanía dándole nuevos significados y contextos de reflexión a la misma. La apuesta se realizaba de la siguiente manera:

Desde tres dimensiones básicas: una filosófica, que apunta al sentido ético-moral de la educación; otra, psicológica, que permite analizar el desarrollo de la personalidad moral de los sujetos y la construcción de sus juicios; y, la tercera, la político-cultural, que permite un acercamiento a las representaciones y prácticas cotidianas de los sujetos (Línea Educación en Valores, 2008, p. 3). 
Aspecto sustancial en la medida que ha propiciado el reconocimiento de la educación ética y moral de los sujetos en pos de transformar referentes valorativos sobre la sociedad en estas dimensiones.

Al ser proyectos de práctica pedagógica investigativa articularon la investigación formativa, la práctica, la experiencia y la reflexión sobre la escuela, todo esto realizado desde una reflexión de largo aliento sobre la presencia de la diferencia y sus reclamos en la sociedad. Por ende, aunque el objeto de conocimiento de los proyectos fue el propio proceso de aprendizaje y formación profesional de los practicantes, así como el ámbito de la enseñanza de las ciencias sociales (aspectos que no serán abordados en este artículo), no podemos dejar a un lado los enunciados, las prácticas y las exigencias que diversos sectores poblacionales (bisexuales, indígenas y estudiantes con necesidades educativas especiales) le hacen a las escuelas. En los documentos finales quedaron plasmados discursos, estrategias y prácticas que demandaban cambios a las instituciones en diversos sentidos; son algunos de estos elementos los que serán retomados en este artículo.

La tercera investigación es la Caracterización de la educación y propuesta para el diseño de un plan estratégico educativo para el Departamento del Chocó, coordinada por Alejandro Álvarez y auspiciada por la Secretaría de Educación del Chocó y la Unicef. En el 2010, este ejercicio recogió información de las instituciones educativas cercanas a la ciudad de Quibdó, capital del departamento en mención (es necesario primero agradecer a Alejandro Álvarez la invitación a participar en el mismo, así como aclarar que para este artículo solo fue utilizada la información que se encuentra en el informe final de la caracterización).

La información de primera mano y el análisis de documentos oficiales e informes suministrados por la Secretaría de Educación del Chocó, facilitaron un acercamiento a realidades educativas rurales periféricas que se repiten en la ruralidad de nuestro país e interpelan la educación formal con retos de hondo calado.

Este análisis llamó la atención sobre múltiples ámbitos, pero en este ejercicio nos centraremos en las particularidades que imponen la ruralidad, la pobreza y la violencia a las instituciones educativas del departamento. Aspectos evidentes en la línea de base educativa construida para el departamento del Chocó. En la misma, fueron evidentes múltiples solicitudes de poblaciones afrocolombianas e indígenas recabadas, analizadas y apoyadas por diferentes organizaciones públicas y privadas que hacen presencia en la región; son estas redes las que soportan lo que más adelante llamaremos nuevos movimientos sociales. 
La cuarta y última investigación se desarrolló en la institución educativa SaludCoop Norte desde el año 2010 hasta el 2013, la cual a la par del proyecto Democracia, Ciudadanía y Derechos Humanos planteó sistematizar información sobre la participación de diferentes entes en el gobierno escolar. La necesidad de analizar de manera general el ámbito educativo cada día se hizo más latente y la óptica de las tensiones, desarrolladas en el último apartado, permitió interpretar algunos fenómenos presentes en la educación colombiana. El gobierno escolar mostró las pugnas por el poder de manera explícita en la configuración de la escuela. En el presente artículo se parte de la idea según la cual el poder es fructífero: "lo que da estabilidad al poder, lo que induce a tolerarlo, es el hecho de que no actúa solamente como una potencia que dice 'no', sino que también atraviesa las cosas, las produce, suscita placeres, forma saberes, produce discursos" (Foucault, 1992; citado en Fanlo, 2007, p. 2). A su vez, Foucault (2006) deja en claro el papel del poder y de los discursos:

Los cuales no se aplican pura y simplemente como una obligación o una prohibición a quienes 'no lo tienen', los invade, pasa por ellos y a través de ellos, se apoya en ellos, del mismo modo que ellos mismos, en su lucha contra él, se apoyan a su vez en las presas (léase fuerzas) que ejerce sobre ellos (p. 64).

Asi mismo, evidenció dinámicas y enunciados que clarifican tensiones presentes en los ámbitos educativos. Así emergieron tensiones entre categorías tales como rural/urbano y alternativo/hegemónico ya que los espacios de pugna en la educación formal configuraban un campo de lucha por privilegiar discursos y ocultar otros; en la misma disputa se les da validez a unos discursos y prácticas en detrimento de otros. La jerarquía lograda en la disputa termina forjando múltiples tipos de participación.

Raymond Williams nos plantea leer la hegemonía cultural como lo hace Gramsci, entendiéndola como una manifestación de la noción de dominación que influye en todos los ámbitos humanos a punto de sustituir la identidad e instaurarle una dominante, ayudándonos a entender lo siguiente:

La hegemonía es siempre un proceso y desde una perspectiva analítica, no es un sistema o una estructura. Es un complejo efectivo de experiencias, relaciones y actividades que tiene límites y presiones específicas y cambiantes. En la práctica, la hegemonía jamás puede ser individual (Williams, 2003, p. 134). 
Así, para cuando habla de la hegemonía en la educación, plantea que:

La educación transmite las habilidades y el conocimiento necesarios, pero siempre a través de una selección particular de la totalidad de la esfera aprovechable y junto con actitudes intrínsecas, tanto para las relaciones sociales como educacionales, que en la práctica son explícitamente incorporativas (William, 2003, p. 140).

Así las cosas, entenderíamos por alternativo todo aquello que interpela, lo que cuestiona, esa "selección particular" que tiene la escuela formal.

\section{Movimientos, visibilidades, discursos y debates}

Los hallazgos irán apareciendo según los sujetos o poblaciones llamados para mostrar su interpelación a los ámbitos escolares. La información de cada una de las investigaciones será retomada buscando tensiones. Esta ordenación busca darle un lugar a los sujetos, ya que son ellos quienes con sus prácticas y discursos transforman la realidad que en gran medida los supera. Utilizamos la categoría de nuevos movimientos sociales porque proporciona un marco flexible pero claro en cuanto los grupos sociopolíticos con los que trabajamos aquí.

Entendiendo por nuevo movimiento social los grupos humanos que actúan sociopolíticamente muchas veces sin un estatuto u organización clara, pero sí, como lo plantea Touraine, con intereses colectivos que se oponen a condiciones impuestas por un adversario que no les son favorables y, así, claramente "cuestionan orientaciones generales de la sociedad" (1997, p. 100). Este tema ha contado con múltiples análisis además de los realizados por Touraine y Santos (ver los estudios de Scott (1990), Dalton y Kuechler (1992), Laranjeira (1990), et al.); empero, aquí más que una definición retomamos el sentido de la lectura que comparten Alan Touraine y Boaventura de Sousa Santos para quienes "la novedad más grande de los NMS (Nuevos Movimientos Sociales) reside en que constituyen tanto una crítica de la regulación social capitalista como una crítica de la emancipación social socialista tal como fue definida por el marxismo" (Santos, 1998, p. 315). Por lo anterior no encontramos que se circunscriban a partidos políticos o estructuras clásicas, pero siguen presionando al poder político mediante reivindicaciones concretas o propuestas alternativas a las hegemónicas. Son estas propuestas o demandas las que los agrupan sin que ello signifique una unidad ideológica compacta. 


\section{Lo afrodescendiente}

Las reivindicaciones de este grupo, evidentes en dos de las investigaciones, empiezan por solicitar reconocimiento ante el ocultamiento histórico padecido. En los procesos consultivos generados sobre los problemas de las comunidades rurales del departamento del Chocó emergieron múltiples "organizaciones comunitarias que impactan las prácticas pedagógicas de las escuelas [y] no luchan exclusivamente por el reconocimiento étnico, su trabajo tiene un enfoque integral. Por ello buscan compartir una problemática generalizada en todos los espacios" (Álvarez, 2010, p. 62).

Así mismo, los movimientos afrocolombianos con ejercicios como la Cátedra de Estudios Afrocolombianos, instauraron desde lo jurídico-pedagógico una apuesta por una educación multicultural que reconozca su legado en la sociedad colombiana. La cátedra de Estudios Afrocolombianos es una reivindicación del movimiento social afrocolombiano "(...) que busca superar el desconocimiento del aporte significativo de los afrocolombianos a la construcción de lo nacional en lo material, lo cultural y lo político (...)" (MEN, 2001, p. 31). Es un instrumento consagrado en el artículo 39 de la Ley 70 de 1993.

Es precisamente esa negación cultural lo que ha vuelto más necesaria una educación propia o etnoeducación, tal como lo hizo el Palenque de San Basilio y posteriormente hicieron los raizales, poblaciones que lucharon vehementemente por ser reconocidas y tenidas en cuenta en el marco educativo nacional.

La etnoeducación, como la comprendemos en Colombia en el discurso oficial, está en el artículo 55 de la Ley 115:

Definición de etnoeducación. Se entiende por educación para grupos étnicos la que se ofrece a grupos o comunidades que integran la nacionalidad y que poseen una cultura, una lengua, unas tradiciones y unos fueros propios y autóctonos. Esta educación debe estar ligada al ambiente, al proceso productivo, al proceso social y cultural, con el debido respeto de sus creencias y tradiciones (Congreso de Colombia, 1994).

El palenque es un pueblo fundado por esclavos libertos. Por ello sus reivindicaciones son antiguas, como lo muestra Friedemann (1987): 
Los maestros hasta hace muy poco tiempo no habían tenido informes de que la manera como los niños hablan no era un 'mal castellano' sino su idioma materno, (...). Y en las clases de historia, al igual que en muchas de las escuelas de Colombia, nunca se ha incluido la dimensión de la participación del negro en la formación del país (p. 18).

Los habitantes del palenque aceptaron que necesitaban una educación propia para defender su lengua y su patrimonio cultural, por ello desarrollaron prácticas etnoeducativas, incluso antes del boom producido por la Constitución del 91 y la Ley 70 del 93.

El Palenque de San Basilio es la denominación para el pueblo afrodescendiente habitante del Archipiélago de San Andrés, Providencia y Santa Catalina:

Defiende sus costumbres, prácticas religiosas y su lengua creole [que] se constituyen en características específicas y propias de esta etnia. Sobre este grupo étnico, se han emitido sentencias de la Corte Constitucional (454/99 y 530/99) que reconocen y protegen sus particularidades culturales y procuran la garantía de sus derechos (Presidencia de la República, 2008, p. 13).

Otra demanda tiene que ver con la toma de conciencia como población segregada históricamente, tanto económica como culturalmente. La primera es evidente en la ubicación territorial de estas poblaciones; las regiones que habitan son las más apartadas e inaccesibles de la nación. La segunda, tiene que ver con el usufructo de la riqueza al no disfrutar plenamente de sus derechos sociales, económicos, políticos y culturales; la riqueza es sacada tanto del territorio como de la población que lo habita. Aunque no es nada nuevo que la explotación de los recursos naturales en su gran mayoría no deje beneficios para las comunidades asentadas allí; lo sorprendente es que el marco constitucional de defensa de derechos continúe sin ser aplicado cuando han pasado más de 24 años de instaurado. Por ello, las comunidades continúan apelando a la educación para conocer sus derechos y hacer factible su defensa.

La investigación mostró que la población no cree en las políticas gubernamentales porque las ven alejadas y relacionadas con intereses centrales que niegan el contexto. 
El informe identifica una falta de credibilidad en las administraciones educativas del orden local, departamental e incluso nacional, se reconoce lo inadecuado de muchas de las políticas y las medidas que se toman en la gestión del sistema educativo, se evidencian los malos resultados y se conocen las causas y sus efectos (Álvarez, 2010, p. 32).

Lo cual va de la mano del racismo soterrado persistente en Colombia y negado constantemente (Jiménez, 2006). Argumentos autojustificantes de los racistas es la autosegregación, la cual niega que esta realidad obedece a procesos históricos que los obligaron a permanecer en espacios específicos y periféricos de la nación para sobrevivir. Aún es visible cómo su presencia en el centro de la nación es objeto de burla y de un racismo soterrado que los excluye.

En varias escuelas fueron evidentes los casos de racismo latentes en formas lingüísticas utilizadas en el trato con los afrodescendientes "(los apodos, burlas, chistes y formas de exposición social los vuelven vulnerables y los exponen a la sanción social)" (Álvarez, 2010, p. 32). La investigación reconoció la presencia de prejuicios racistas que deben ser atendidos por los docentes, tal como lo manifestara la profesora Cecilia, en una entrevista del 2004:

Si tú no les paras bolas, cuando están pequeños, ya cuando grandes no les quitas el racismo por nada, ni con nada. Tiene que ser cuando están pequeños. El currículo en sexto y séptimo permite hablar de ello, después es más difícil (Jiménez, 2006, p. 26).

Los docentes que luchan por cambiar los estereotipos que sustentan estas prácticas luchan contra el racismo construido como diferencia cultural pero sustentada colectivamente en que hay una diferencia biológica. "Al ser soterrado, indirecto, camuflado este racismo instaura estereotipos negativos de esta población" (Jiménez, 2006, pp. 67-68), establecen dinámicas simbólicas violentas que reproducen los afrocolombianos. Por ello, el movimiento afrodescendiente realiza demandas al sistema educativo por una lucha formal contra estos estereotipos mediante prácticas pedagógicas que reconozcan lo siguiente:

(...) La demanda de respeto a su diferencia étnica en el marco de la interculturalidad. En esta última es donde se reconoce la importancia de trabajar en una apuesta por una educación intercultural (Rojas y Castillo, 2006; MEN, 2004; Villa y Grueso, 2008; Cassiani, 2007) como proyecto que reformule aspectos 
epistémicos, políticos, éticos y sociales, para enriquecer la convivencia de múltiples culturas y especificidades (Jiménez, 2011, p. 95).

Otra reivindicación es la solicitud de ser sacados del conflicto armado y territorial del país. Gran parte de la población de afrodescendientes habita territorios disputados por grupos guerrilleros, paramilitares y narcotraficantes que los convierten en víctimas constantes de amenazas, desplazamiento y asesinato. La violencia que impone la guerra destruye su relación con el territorio e impone dinámicas de desarraigo refirmados por la violación de los derechos de estas poblaciones, en algunos casos con complicidad de las fuerzas militares colombianas, como lo demostró la desmovilización de las autodefensas. "La respuesta de estos grupos fue realizar comunidades de paz en donde el componente educativo ha sido fundamental para difundir y defender sus derechos" (Bernal y Jiménez, 2011).

\section{Lo indígena}

Las 80 comunidades indígenas de nuestro país comparten fenómenos, características y territorios con los afrocolombianos; por lo tanto, también realizan las mismas demandas que hace el movimiento afrodescendiente aunque desde un marco distinto. Al ser movimientos reivindicativos desde lo étnico los ubica en un lugar similar aunque no igual; los indígenas soportan su defensa de derechos étnicos anterior a la constitución de 1991, mientras la gran mayoría de los movimientos afrocolombianos surgen posteriormente a la Constituyente de 1991.

La lucha indígena es titánica, ellos han defendido su cultura ubicándose en oposición a la cultura occidental del "blanco mestizo", configurándose como fuerza contestataria que por dinámicas históricas de represión, olvido y segregación ha sido reprimida. Aquí es necesario aclarar que existen movimientos organizativos de importante calado como el logrado por las luchas de los inicios del siglo XX en el Cauca, la Sierra Nevada de Santa Marta, en el Huila, en Caldas y en el Tolima. Sin embargo, la violencia bipartidista cobró su cuota y desarticuló la mayoría de las organizaciones indígenas. Posteriormente ha sido el Consejo Regional Indígena del Cauca - CRIC y su lucha desde el 70, quienes han logrado la formación de la Organización Nacional Indígena Colombiana - ONIC, que reúne a más de 41 organizaciones.

Esta razón la lleva a ser uno de los movimientos sociales más importantes del país. Ejemplo de ello son las mingas indígenas generadas en el 2004, en el 2008 
y en el 2011; con movilización de más de 12 mil personas marchando por sus derechos en diferentes ciudades y carreteras del país. Estas movilizaciones también se han manifestado en contra de la guerra y el conflicto armado de nuestro país, generando que tomen distancia de los actores del conflicto. Los indígenas han superado el miedo a la movilización y han solicitado vehemente el retiro de todo actor armado de sus territorios, incluyendo al Ejército Nacional. Conflicto que las Naciones Unidas denomina como la peor crisis humanitaria en el hemisferio occidental. El mismo lleva consigo abusos y violaciones a los derechos humanos por el desplazamiento forzoso de aproximadamente cuatro millones de colombianos, muchos de ellos pobladores indígenas.

La violencia estructural ha ido de la mano de la violencia simbólica, pues hay manifestaciones culturales de segregación y exclusión para con los indígenas. Por ello la reivindicación de tierras es fundamental para estos movimientos. Por un lado está el ejercicio de visibilización, sino que, además, el reclamo de tierras permite que ellos actúen en concordancia con su cosmología. "Aunque los discursos y las acciones por el reconocimiento han logrado visibilizarlos en el panorama nacional, las condiciones educativas de las comunidades en los territorios aún siguen siendo más que deficientes o inexistentes" (Álvarez, 2010). Por esta razón, los movimientos indigenistas reivindican un discurso de reconocimiento y respeto nacional a su visión alternativa al modelo de desarrollo occidental.

Las paupérrimas condiciones de la ruralidad de Colombia están de la mano de una lectura histórica, donde el desplazamiento y la confinación a zonas de difícil acceso han sido constantes. Por ello a que las comunidades indígenas le hayan apostado al distanciamiento y dispersión espacial como esquema de defensa cultural. Esto demanda retos logísticos y pedagógicos de extrema complejidad a la educación formal, en especial por el acceso tanto para docentes como para estudiantes, sin contar con los contenidos que se deben y pueden difundir en estas instituciones. El gobierno reconoce que los recursos son insuficientes para atender las necesidades tanto pedagógicas como físicas que impone una dispersión tan alta de las comunidades, como lo muestra Álvarez (2010) en la entrevista con la rectora:

Son evidentes dos grandes necesidades en lo que se refiere a las condiciones físicas de las escuelas indígenas de la región: una, su mejoramiento; y dos, la adecuación pedagógica a las necesidades que plantean las comunidades. Para ello, en muchos documentos se plantea la necesidad de poseer recursos para la consolidación de los espacios educativos en cada una de las instituciones indígenas. 
Aunque los recursos que llegan por medio de transferencias a las propias comunidades son invertidos en la adecuación de infraestructura para las sedes 0 institutos indígenas, es claro que no es suficiente (p. 64).

Estas limitaciones han impulsado la urbanización de las comunidades para acceder a instituciones educativas. Empero, esto plantea otro problema, ya que la convivencia con comunidades que no respetan ni valoran su diferencia cultural, e incluso llegan a denigrarla, genera mayor exclusión. Por ello se ha vuelto una constante la solicitud de enfoques específicos por comunidad en los cuales se defienda y respete la diversidad cultural de los indígenas. Ejemplos hay muchos, pero es diciente que la plataforma de lucha que han establecido busque una educación bilingüe y bicultural bajo el control de las autoridades indígenas. En especial porque se hacen manifiestos los conflictos entre los enfoques indígenas y los enfoques homogenizantes de occidente. "No olvidemos el énfasis occidental impuesto desde lo blanco y lo mestizo" (Álvarez, 2010).

La articulación de saberes indígenas con el territorio realizado en el Colegio Psicopedagógico Villaverde fue apoyado por la Corporación Casa Asdoas, en la localidad de Usme de la ciudad de Bogotá, abordando problemáticas ambientales en dicha localidad. "El registro de información estuvo de la mano de las prácticas pedagógicas que resignificaron y defendieron el territorio" (Rico, 2010). Ello sin duda demanda trabajos de largo aliento en donde los agentes educativos construyan nuevos marcos de enunciación que puedan ser avalados por la institucionalidad imperante en la educación formal, sin que esto implique que el marco indígena deba insertarse en lógicas jerárquicas, de desarrollo lineal, jerarquización y competencia; ya que de esta manera negarían los marcos epistemológicos de donde surgen las propuestas.

Otra dificultad es el tipo de educación que imparte el sistema educativo, el cual termina siendo muy directivo y alejado de preceptos pedagógicos que en el momento están siendo discutidos por los movimientos indígenas. "Por ello la apuesta por formar profesionales indígenas como puente entre los contenidos y saberes de Occidente y los saberes y apuestas propias" (DAPI, 2008, pp. 69-70). La apuesta por la educación propia permite la reproducción de costumbres y de su lengua, aspecto sustantivo como comunidad.

La lucha por no ser segregados tanto económica como culturalmente tal como las minorías soslayadas por el discurso pro-mestizo, católico y jerárquico, las ubica en la resistencia y la lucha por sus derechos civiles, económicos y culturales reconocidos en la Constitución de 1991, dándoles la condición de los movimientos 
indígenas que hemos destacado. Sería ingenuo de parte nuestra no reconocer que el discurso de nación instaurado en la educación aún sigue ocultando disimuladamente las minorías étnicas presentes en el país. Por ello los ejercicios de visibilización sociopolítica en búsqueda de lograr un posicionamiento donde sus discursos logren una mayor aceptación.

\section{Lo ambiental}

Es importante ver cómo lo abordado referencia lo ambiental tanto en los discursos de los indígenas como en los enunciados de los afrocolombianos. Sin embargo, este discurso tiene una masa crítica que ha generado conciencia frente al modelo de desarrollo expansionista y extractivo que defiende occidente. Las críticas a las llamadas "locomotoras de desarrollo" propuestas por el gobierno de Álvaro Uribe Vélez y continuadas por Juan Manuel Santos ha sido catalogado de economicista y poco responsable por los movimientos ambientalistas que critican fuertemente el impacto que estos proyectos tendrán sobre el entorno.

Desde los años setenta, diversas ONG (la Fundación Herencia Verde, Cosmos, entre otras) y grupos locales (Consejo Ecológico de la Región Centro Occidental CERCO, Grupos Ecológicos de Risaralda - GER, etc.) trajeron nuevas perspectivas en torno a lo ambiental, las cuales han interrelacionado la conservación, los problemas sociales y protestas en contra del desarrollo. "Esta mirada social ha permitido la interacción de estas organizaciones y grupos ambientales con las demandas sociales, económicas y políticas de los movimientos de indígenas, campesinos y afrocolombianos" (Ulloa, 2001, p. 11). A su vez los discursos de respeto por lo ambiental han llevado a afrodescendientes e indígenas a convertirse en guardianes del medio ambiente. Incluso hay organizaciones indígenas que han ganado premios a nivel mundial por su trabajo de defensa del medio ambiente; podemos mencionar a los Embera, los U'wa y los Kogui. Lo interesante de estos movimientos es que impactan la construcción de identidad nacional porque empiezan a circular nuevas formas de identificarse y explicar la existencia, al punto de impactar políticas culturales nacionales, como lo muestra Ulloa (2001):

De esta manera, los indígenas "usan" su identidad colectiva como una estrategia performativa para establecer relaciones (políticas, económicas, etc.) con el Estado (Gros, 1999), y como una estrategia política que les permite el manipular su situación histórica y cultural. La identidad colectiva se convierte en una 
construcción histórica que les permite a los movimientos indígenas luchar por sus intereses políticos en el ámbito nacional e internacional, como ocurre con las identidades ecológicas (pp. 7-8).

Estos ejercicios de reivindicación están ligados con la identidad y han impactado la educación formal; no es gratuito que en la mayoría de los colegios se trabaje y valore el Proyecto Ambiental - PRAE (a nivel jurídico la integración de estos discursos está en decretos como el 1743 de 1994). Una de las tesis guiadas en la Línea de Educación en Valores trabajó la eco-pedagogía y la apropiación territorial como elementos básicos en la formación para una convivencia de los jóvenes. Su apuesta fue mostrar cómo los jóvenes, mediante un proceso pedagógico, se apropian del territorio, reconociendo el pasado del mismo y sus actuales condiciones ambientales proponiendo nuevas formas de leer el territorio. El docente-investigador Carlos Rico en su proyecto pedagógico articuló la formación ciudadana y los referentes de identidad indígenas desde lo ecológico con jóvenes del Colegio Psicopedagógico Villaverde, en el marco del servicio social. Son actividades que deben desarrollar los jóvenes en su comunidad y que está reglamentado por la Ley General de Educación 115 de 1994, en su artículo 97. Sin olvidar el Decreto 1860 en su Artículo 39 donde se define como "la integración a la comunidad para contribuir al mejoramiento social, cultural y económico (...)".

Este ejercicio afectó no solo el espacio en mención, sino la malla curricular del colegio al integrar problemáticas ambientales a la formación de los estudiantes con el objetivo de hacerlos críticos frente a la clásica relación de occidente con el ambiente. Tan pronto como se propusieron nuevas formas de relación con el territorio, a partir de los saberes ancestrales de los indígenas, el referente ecológico de protección del Río Tunjuelo emergió con potencia. La territorialidad en este estudio fue tomada como el conjunto de relaciones tejidas por el individuo en tanto que es miembro de una sociedad, con su entorno. Algunos autores como Malmberg (1984) "consideran importante tener en cuenta que la territorialidad no solo habla del vínculo de los grupos sociales con su entorno, sino que expresa que este vínculo también incluye un componente de tipo emocional entre los individuos y su espacio" (Ramírez y Aguilar, 2006, p. 14).

"La visión indigenista se integró a las exigencias exógenas de las autoridades de vigilancia y control educativas, así como a las necesidades y expectativas de la comunidad y contexto" (Rico, 2010), haciendo que los sujetos participantes transformaran su marco axiológico; anteponiendo valores contrapuestos a los defendidos 
por el paradigma desarrollista y progresista, hegemónico en la educación formal colombiana.

\section{La pro-democracia}

Esta categoría provisional intenta relacionar, parcialmente, múltiples demandas que están vinculadas con movimientos más específicos, como son los grupos de identidad sexual y las poblaciones con necesidades educativas especiales, entre otros. Estas demandas han aparecido en las investigaciones analizadas en este artículo y requieren mayor reflexión para comprender los órdenes discursivos y su sentido en el ámbito educativo. Así las cosas, presentaremos un paneo general de esquemas articuladores de los nuevos movimientos sociales que pugnan por la participación y la justicia como aspectos cardinales para hablar de democracia en la escuela.

La participación es el interés más evidente en los discursos y las acciones de los nuevos movimientos sociales aquí mencionados; el segundo reclamo está asociado con la justicia entendida como equidad y no solo como el respeto acrítico de un marco jurídico. La participación se entiende aquí como la define Sartori (1994):

Es tomar parte personalmente, un tomar parte activo que verdaderamente sea mío, decidido y buscado libremente por mí. Así no es un 'formar parte' inerte ni un 'estar obligado' a formar parte. Participación es ponerse en movimiento por sí mismo, no ser puesto en movimiento por otros (pp. 74-75). Estas dos búsquedas ordenan el uso argumentativo de los nuevos movimientos sociales en lo democrático. Las constantes solicitudes de espacios de participación como espacios de incidencia buscan orientar de otra manera las prácticas educativas.

La mayor parte de los movimientos aquí mencionados están influidos por discursos en boga a mediados del siglo pasado. Discursos como el del desarrollo son los que más han fomentado la noción de democracia dándoles posibilidades y limitantes. Lo reconoce María Isabel Heredia Duarte (2012) en su investigación de maestría:

El campo de la educación ha sido interpelado por el discurso sobre el desarrollo, entrecruzándose con un campo de concomitancia como lo era el discurso sobre la transformación (o modernidad como técnicamente se le conoce), teniendo en 
cuenta que el desarrollo mismo sugiere a su vez modernidad o transformación constante, los enunciados educativos permanecieron atrapados en esta lógica, que era el principio de verdad mayor o imperante (p. 20).

"Conste pues que el discurso técnico de la modernidad es visible no solo en la dualidad de la educación tanto como servicio y a su vez como derecho" (Heredia, 2012) instaurada en nuestro país. Adicionalmente, este discurso lógico lineal y desarrollista está presente en los currículos que guían la escuela y es su lugar, es el espacio donde más resistencia se realiza. No es gratuito que en varias tesis de la Línea de Educación en Valores de la Universidad Pedagógica, así como en la investigación realizada en el Colegio SaludCoop Norte, el discurso de las competencias laborales y ciudadanas haga presencia regulando saberes y formas de relación políticas dentro de la institución.

Las transformaciones vividas por el campo educativo en la segunda parte del siglo XX permitieron la instauración de un discurso modernizante de la educación en el país. Entendido como modelo etnocéntrico en el cual todas las sociedades deberían ir hacia las sociedades industriales occidentales remplazando su organización social, su visión de mundo, su cultura y su personalidad en búsqueda de una mayor producción per cápita modificando la economía tradicional hacia una más compleja. "Lo que lleva interno un concepto evolutivo, difusionista, jerárquico y sin duda colonialista" (Barfield, 2007, p. 360).

"Este discurso basado en el pragmatismo de la formación de hombres y mujeres capacitados para vivir en un mundo globalizado, cuyo rasgo distintivo es el modelo de producción capitalista" (Calderón y Echeverría, 2010, p. 11).

Este proceso visible en otros trabajos de grado guiados (Sierra, 2010) muestra cómo los Proyectos Educativos Institucionales -PEI- han cambiado para estar acorde con el discurso sobre las competencias. Los marcos interpretativos en estos cambios siguen ligados al concepto de modernización, haciendo que los espacios escolares inserten el discurso del desarrollo como un objetivo incuestionable. Este concepto sin lugar a dudas va unido a los discursos multilaterales:

El medio ambiente, la justicia social, la democracia, la educación y el intercambio de conocimientos están estrechamente vinculados al desarrollo, (...) ni la democracia política puede consolidarse sin medidas económicas y sociales que propicien el desarrollo ni una estrategia de desarrollo puede prosperar sin la legi- 
timidad y el refuerzo que le confiere la participación democrática (Boustros-Ghali, 2003, pp. 10-11).

Así, la educación termina configurada como un campo de disputa en donde los discursos sobre la modernidad y el desarrollo lograron ser hegemónicos. Lo más complicado es que este predominio discursivo no plantea una visión alternativa a los problemas que la modernidad con sus contradicciones instauran en la vida social y ambiental.

Conviene advertir que el discurso de la participación democrática ha estado acompañado de las demandas por un marco jurídico estable basado en la justicia liberal occidental; es decir, la justicia liberal es comprendida como requisito sine qua non para garantizar el ejercicio democrático e instaurando un solo tipo de jurisprudencia. Aunque reconozcamos que el liberalismo no tiene una posición unitaria, sí podemos identificar tres principios básicos en su concepción de justicia: el universalismo, el individualismo ético y la neutralidad. Lo que lo lleva a verse "a sí mismo, por tanto, como una doctrina universal capaz de trascender culturas y fronteras apelando exclusivamente a la razón humana" (Aguilar, 2003, p. 3). La justicia occidental promociona e instaura aspectos como el racionalismo, la noción de igualdad, la individualización, la noción de derecho natural, la concepción de libertad (individual y de propiedad), la limitación del poder general, el juego del mercado y la búsqueda acumulativa.

El mayor problema de la anterior mirada es su incomprensión:

Ya que la justicia es absolutamente dependiente de cada cultura (...) [y reconocer esto nos pone ante] dos estrategias de evasión: o se declara ingenua la idea de justicia, en todo caso justificable por la experiencia común y, como lo hacen muchos investigadores del derecho y de la sociedad, se elige renunciar a ella, o se abusa de ella a favor de los propios intereses (Höffe, 2007, p. 57).

El marco neoliberal, al defender una sola visión, tambalea cuando se habla de reivindicaciones culturales ubicadas en otro lugar de comprensión del mundo. ¿Qué es justo para los indígenas, para los afrocolombianos?, ¿cuál referente de justicia defendemos en el ámbito formal de una educación que los tenga en cuenta? Es allí donde aparecen discursos sobre la justicia desde diferentes ámbitos.

Otras investigaciones muestran los reclamos de justicia realizadas desde el interior de los movimientos de maestros. Estos movimientos sociales comprenden 
que tienen una participación restringida o injusta en la esfera pública de la educación. "Un elemento de constantes referencias y asumido como una situación de injusticia, es la participación sumamente restringida como sujetos activos de política, percibiéndose por parte del Estado solo como objetos de la misma" (Cogollo, 2011, p. 114). Lo cual lleva a que la injusticia que ellos perciben se traslade a ámbitos más específicos, siendo posible verla en las percepciones tanto de los estudiantes como de los padres dentro del Gobierno Escolar del Colegio SaludCoop Norte. Para estos sujetos los espacios de participación, al ser tan amplios y complejos, terminan negando la misma participación, y cuando esto ocurre el sujeto se resiste a participar. Al ver que solo sería efectiva su participación si conoce al dedillo la norma o la lógica interna del proceso. Sea como fuere, el proceso va perdiendo legitimidad ante los sujetos al ser tan amplio y va difuminando la posibilidad de intervenir y decidir de los mismos.

Podríamos decir que este fenómeno configura de facto una injusticia que niega el derecho a la participación. Por ello la sensación de distancia de los sujetos ante la democracia participativa o representativa, ya que, tal como está estructurada, termina diluyendo los discursos y por lo tanto la posibilidad de influir en la lógica hegemónica. Esta misma lógica o discursiva general se va alejando de los sujetos y termina siendo solo parte de saberes técnicos o muy especializados que operan el sistema; que termina perdiendo el sentido colectivo y no los respalda la mayoría.

Un ejemplo es visible en la distancia que toman los estudiantes de instancias como el Consejo Estudiantil:

¿Para qué participo en el gobierno escolar? Si cada vez que lo hago hay reuniones en donde se discute tanto que no se llega a nada, y si se llega es a lo mismo que las coordinadoras o la rectora quieren (estudiante de grado once, Investigación Democracia, Ciudadanía y Derechos Humanos).

Este fenómeno muestra la apatía, desconfianza y baja participación de los ciudadanos colombianos en los espacios de decisión y fiscalización.

En dos ejemplos adicionales es factible de observar tanto las reivindicaciones de grupos de identidad sexual como las reivindicaciones de poblaciones con necesidades educativas especiales. En cuanto los primeros, análisis de Sierra (2010) nos permiten reconocer que lo que antes era normal está cambiando, y que los discursos reivindicativos desde la identidad sexual se hacen presentes con movimientos en pro de los derechos de las lesbianas, gays, bisexuales, transgeneristas 
e intersexuales (comunidad LGBTI). En especial porque la escuela aún tiene el marco histórico "de un discurso machista, heterosexista, católico y patriarcal, que solo concibe la heterosexualidad como única forma posible de interactuar sexualmente con los otros" (Sierra, 2010, p. 46). Es evidente cómo este discurso trata de "corregir" toda aquella "desviación" sexual que no esté acorde con la visión hegemónica de la heterosexualidad. La institucionalidad como reproductora de un discurso seguirá presentando una resistencia a estas reivindicaciones de derechos, y los nuevos movimientos sociales seguirán reivindicando sus derechos sexuales. Aunque hay muchos más ejemplos a nivel nacional e internacional que deben ser tenidos en cuenta en un análisis de esta manifestación de la diferencia sexual al interior de la educación formal (Mejía y Almanza, 2010), baste con la mención hasta aquí realizada para ver cómo la diferencia de género también interpela el discurso hegemónico escolar.

Otros discursos que han impactado la escuela son los que pugnan por una educación que reconozca, integre y respete las poblaciones con necesidades educativas especiales. Estos discursos también han impactado el orden discursivo jurídico (Decreto 366 de 2009) generando desde allí enunciados que defienden la integración y una educación inclusiva. Fenómeno poco sencillo que ha demandado a las instituciones realizar ejercicios tanto curriculares como físicos, para atender a esta población tal como lo expresa el artículo cuatro del mencionado decreto:

Los establecimientos educativos que reporten matrícula de estudiantes con discapacidad cognitiva, motora, síndrome de Asperger o con autismo deben organizar, flexibilizar y adaptar el currículo, el plan de estudios y los procesos de evaluación de acuerdo a las condiciones y estrategias establecidas en las orientaciones pedagógicas producidas por el Ministerio de Educación Nacional (1999, Artículo 4, Decreto 366).

Aspecto visible en la investigación del Colegio SaludCoop Norte como en la investigación de Gómez (2012). Esta segunda investigación fue realizada en un colegio que tiene integrada la educación especial a sus objetivos institucionales.

Podemos decir que en las exigencias antes mencionadas es visible luchas tanto por una justicia más equitativa como por una participación más allá de la democracia representativa que rige el ámbito escolar hasta hoy. Además, surgió que estas reivindicaciones claramente son usadas por movimientos los cuales desde la diferencia están cambiando la educación formal en nuestro país. Estos nuevos 
movimientos sociales defienden enunciados de grupos en otrora ocultos o negados, piden una nueva educación, una relectura de la diferencia.

\section{Las tensiones y sus preguntas}

El artículo se organizó para mostrar los reclamos que realizan ciertos grupos sociales que al tener intereses conjuntos, se catalogan como movimientos sociales. Estos reclamos los hemos comprendido como la evidencia de unos discursos que hacen presencia y están en conflicto, recordemos que el conflicto lo entendemos como la tensión existente entre discursos que propenden conseguir una mejor posición frente a la lógica hegemónica en el campo educativo colombiano; siendo lo planteado por Foucault (1982) para la construcción de los discursos sobre el saber al interior del sistema educativo, al punto de exigirle reflexiones profundas para reorientar sus prácticas. Las tensiones que emergen de este conflicto consolidan el ámbito de la educación formal como un campo de saber en dónde los nuevos movimientos sociales hacen presencia en busca de posiciones privilegiadas en la sociedad.

Sin embargo, esta reorientación del quehacer de la escuela encuentra resistencia no solo con otros discursos sino con la misma estructura escolar, ya que la misma no fue construida para desarrollar lo que estos discursos le solicitan.

Es evidente que la información mostrada admite deducir múltiples tensiones, pero en estas conclusiones no profundizaremos en las tensiones que el análisis permite, ya que espacio es bastante limitado. Lo sensato será enunciar las tensiones y plantear lo que implica su emergencia en nuestro contexto, así como las preguntas que nos suscitan.

Una de las tensiones que atraviesan los discursos de los cuatro movimientos sociales identificados en este artículo tiene que ver con una transformación demográfica que nuestra sociedad ha vivido en los últimos años: la urbanización; el fenómeno de la concentración poblacional en las urbes se ha manifestado mundialmente. Esta realidad nos permite ubicar los reclamos trabajados aquí, y establecer cómo emerge la tensión entre lo urbano y lo rural en donde se ubican muchos de los reclamos realizados por estos movimientos. "El proceso de urbanización dejó de ser hace mucho tiempo un mero proceso cuantitativo, de mera acumulación demográfica en torno a una acumulación de recursos, para pasar a ser un proceso de carácter cualitativo" (Baigorri, 1995, p. 3), lo que implica una relación de tensión entre lo rural y lo urbano. 
La lógica urbana es diferente a la lógica rural, sin duda la lógica urbana ha impactado los ámbitos rurales, sin dejar de lado que han existido resistencias a esta influencia. La ruralidad ante los enunciados de las competencias ciudadanas difundidas por los contextos urbanos, por ejemplo, han tenido una cuota muy baja de aceptación y reconocimiento. Otro elemento que alimenta esta tensión es la presencia del Estado, el cual en el contexto rural sigue siendo mínimo o casi inexistente. Ante estas dos realidades los movimientos sociales han propuesto una demanda por la educación propia. Frente a este conflicto Pérez (2014) Manifiesta que es necesario que el sistema educativo se adapta al estudiante reconociendo sus constructos sociales y culturales. Hay múltiples ejemplos de cómo la ruralidad genera cuestionamientos a los valores insertos en la educación formal que viene de lo urbano. Fundamentalmente porque no los tiene en cuenta y propone un modelo de desarrollo y acumulación al que le apuesta el país y sus élites dominantes. Esta tensión nos permitiría pensar en que la identificación cultural termina generando tensiones en todo el sistema educativo, y que la hegemonía del modelo neoliberal aborda de manera muy burda. Si nuestra educación quiere responder a la característica de ser una nación pluriétnica y multicultural, como mínimo deberíamos estar hablando de modelos híbridos (García-Canclini, 2000, p. 8) para los espacios educativos urbanos y rurales con poblaciones mixtas.

Reconocer las concepciones y las prácticas propias en los pueblos ancestrales implica radicalizar aún más la presencia de proyectos educativos contextualizados que, aunque dialoguen con axiomas occidentales que estandarizan, estén en posibilidad de sobrevivir al cuestionarlos porque de ello depende la respuesta a las necesidades que las comunidades. Modelos híbridos que apliquen para las ciudades porque las mismas se han convertido en "laboratorios en los que se desarrollan las formas y los medios para la convivencia humana pacífica y para el diálogo y el entendimiento transculturales" (Bauman, 2008, p. 29).

Sin duda, una tensión que se hace más que evidente en nuestra realidad es la generada entre la guerra y la paz (preferimos hablar de guerra ya que la misma retrata más cómo en nuestro país los conflictos, de diverso orden, han intentado ser solucionados mediante el uso sistemático de las armas). Los fenómenos sociales generados a partir de la presencia de grupos armados en todo el país es una constante que impide los procesos que Amartya Sen ha catalogado como la "expansión de las libertades reales de las que disfrutan los individuos" (2006). No podemos negar que las lógicas que impone el conflicto armado colombiano obedecen más a una lógica moderna 0 , incluso, premoderna que sigue basada en presupuestos 
occidentales de apropiación, uso, explotación y dominación del territorio. Las guerras aunque no son exclusivas de Occidente, en Colombia las mismas han estado unidas a lógicas occidentales en diferentes órdenes de usufructo; pero, además, las clases oligárquicas han negado la diferencia, despreciado el diálogo e imposibilitado el acuerdo. Podríamos preguntarnos, ¿cómo acordar con el diferente si en la guerra, idea que han manejado diversos presidentes, la idea es la integración, la imposición o la eliminación del otro?

Es así como las poblaciones indígenas y afrocolombianas se enfrentan a múltiples problemáticas ambientales (manifiestas en el calentamiento global, la minería irracional y la pérdida de biodiversidad constante), las generadas por el narcotráfico (los cultivos ilícitos, el tráfico de armas y el de dinero) y las que genera el conflicto armado (presencia de grupos armados, enfrentamientos militares y desplazamiento constante). Todas estas problemáticas obligan a una movilidad interna en el departamento que es visible a nivel individual y grupal (Álvarez, 2010, p. 53).

Podemos decir que el conflicto armado y la violencia se han vuelto pan de cada día para la mayoría de las poblaciones rurales, lo que ha transformado principios axiomáticos como la solidaridad, la compasión, la confianza y el respeto por el "otro". La violencia se traga a los sujetos y lo único que escupe es un ser individualista que fomenta el lucro y la acumulación personal en detrimento de su propia sociedad. Ese, sin duda, es el mayor peligro del conflicto armado que vivimos.

Sin desconocer la importancia de concebir lo justo desde lo jurídico, lo cual es necesario para la sociedad occidental, ¿deberíamos preguntarnos por un marco de justicia que reconozca otras reivindicaciones culturales, otros lugares de comprensión del mundo para superar la guerra y apostarle a la paz? Aquí podrían ser útiles reflexiones como las de Ricoeur, quien propone superar la visión occidental de la justicia que la reduce a un marco jurídico racional ya determinado y ubicar la noción de lo justo en otro referente; desde un plano de sabiduría práctica que revise las nociones clásicas de lo bueno o de lo legal. Sería más fructífero hacer referencia a lo equitativo. "Lo equitativo es la figura que revela la idea de lo justo en las situaciones de incertidumbre y de conflicto, para decirlo todo, bajo el régimen ordinario o extraordinario de lo trágico de la acción" (Ricoeur, 2003, p. 37).

La última tensión que identificaremos en este texto será la generada entre lo hegemónico y lo alternativo, y son los nuevos movimientos sociales que son periféricos y alternativos. Las comunidades afrocolombianas, las indígenas, más los otros 
movimientos sociales comparten el interés de no seguir siendo segregadas cultural y económicamente del centro del país. Por ello su pugna por capturar la centralidad y transformar la lógica occidental de negación por una lógica de reconocimiento de la diferencia. "Esta pugna no es meramente discursiva, sino que se vuelve pragmática al exigir normas favorables, recursos financieros y apoyo para la sobreviviencia cultural" (Gutmann, 2008).

Estas tensiones implican múltiples cambios. Uno de los más significativos es que la formación docente debe cambiar, de lo contrario seguiremos fomentando ejercicios de exclusión animados por los marcos valorativos neoliberales. Los ejercicios de formación docente deben ser más contextualizados y que planteen discusiones de hondo calado moral sobre las políticas públicas de estandarización defendidas desde los diferentes Ministerios de Educación latinoamericanos que, en gran medida, responden al modelo neoliberal. Así, las políticas públicas educativas reconocerían que "ninguna cultura puede ser valorada como absolutamente mala o absolutamente buena. Las culturas son necesariamente diferentes unas de otras" (Freire, 2007, p. 123). Requerimos de diálogos significativos en que los acuerdos no estén preestablecidos, en donde estemos dispuestos a cuestionar el modelo de desarrollo establecido por occidente el cual ha demostrado ser insuficiente para nuestra complejidad. Como se deduce de lo ya dicho, los nuevos movimientos sociales cuestionan el modelo de desarrollo y nos ubican en discusiones sobre lo humano, lo tecnológico, lo ambiental y el impacto que dejamos en los territorios que habitamos. Tal vez, reflexionando sobre estas tensiones podamos pensar un nuevo mundo, una educación para humanos en donde la imaginación recoja la diferencia potenciando un futuro colectivo.

\section{Referencias}

Aguilar, F. (2003). Teorías modernas de la justicia. Pontificia Universidad Javeriana. Recuperado de http://javeriana.edu.co/Facultades/C_Sociales/Profesores/jramirez/PDF/AguilarTeorias_de_justicia.pdf

Alguacil, J. (2003). La ciudadanía emergente y sus síntomas. T. Arenillas, Ecología y Ciudad. Raíces de nuestros males y modos de tratarlos, 267-285. Madrid: El Viejo Topo.

Álvarez, A. (2010). Informe: caracterización de la educación y propuesta para el diseño de un plan estratégico educativo para el departamento del Chocó. Colombia: Secretaría de Educación del Chocó - Unicef. 
Baigorri, A. (1995). De lo rural a lo urbano. Universidad de Extremadura. Recuperado de http://www.eweb.unex.es/eweb/sociolog/BAIGORRI/papers/rurbano.pdf

Barfield, T. (2007). Diccionario de Antropología. México: Siglo XXI Editores.

Bauman, Z. (2008). Múltiples culturas, una sola humanidad. España: Katz Editores.

Bauman, Z. (2005). Vidas Desperdiciadas. La Modernidad y sus parias. Barcelona: Paidós.

Bernal, M. D. y Jiménez, C. E. (2011). Reconstrucción de las escuelas en democracia y paz, experiencias y saber pedagógico, 2003-2009. Bogotá: Corporación Podion.

Boustros-Ghali, B. (2003). La interacción entre democracia y desarrollo. Paris: Organización de las Naciones Unidas - UNESCO. Recuperado de http://unesdoc.unesco.org/ images/0013/001323/132343s.pdf

Calderón, A. y Echeverría, J. (2010). Ciudadanía y Convivencia: hacia la construcción de ambientes democráticos dentro de la escuela. Bogotá: Universidad Pedagógica Nacional, LEBESC, tesis de grado.

Calvo, C. (2005). Complejidad, caos y educación. G. A. Arellano Duque, La educación en tiempos débiles e inciertos, 115-136. España: Antrophos.

Cogollo, C. (2011). Transformación de marcos de interpretación y acción política en el nodo Caribe de la plataforma de análisis y producción de políticas educativas, 20042008. Bogotá: Universidad Pedagógica Nacional, tesis de maestría.

Congreso de Colombia. (1994). Ley 115 de 1994. MEN. Recuperado de http://menweb. mineducacion.gov.co/normas/concordadas/Decreto115.htm

DAPI. (2008). Plan de Desarrollo del Choco, 2008-2011. Gobernación del Chocó. Departamento Administrativo de Planeación e Infraestructura (DAPI). Recuperado de http:// choco.gov.co/plan-de-desarrollo-del-choco.html

EPN. (2003). Al encuentro con nuestra cultura afrocolombiana. Bogotá: Universidad Pedagógica Nacional; Fundación Antonio Restrepo Barco.

Fanlo, L. (2007). Emergencia de la matriz militar-discursiva argentina: el discurso de Leopoldo Lugones. Recuperado de http://www.catedras.fsoc.uba.ar/fanlo/fanlo_ejercitoyargentinidad.pdf

Foucault, M. (1991). El sujeto y el poder. Bogotá: Carpe Diem Ediciones.

Foucault, M. (1984). La arqueología del saber. México: Siglo XXI de Colombia.

Foucault, M. (1982). Las palabras y las cosas. Bogotá: Siglo XXI Editores.

Foucault, M. (2006). Vigilar y castigar. Bogotá: Círculo de Lectores.

Freire, P. (2007). Pedagogía de la tolerancia. Buenos Aires: Editorial Fondo de Cultura Económica; Centro Regional para la Educación de Adultos en América Latina y el Caribe (CREAL).

Freire, P. (2005). Pedagogía del oprimido. México: Siglo XXI. 
Friedemann, N. S. (1987). Ma Ngombe. Guerreros y ganaderos en Palenque. Bogotá: Carlos Valencia Editores.

García-Canclini, N. (2000). La globalización, iproductora de culturas hibridas? Recuperado de http://www.slideshare.net/jcarangoq72/ ernesto-garcia-canclinila-globalizacin-productora-de-culturas-hbridas

Gómez, S. (2012). Propuesta para el mejoramiento de la convivencia de la institución educativa distrital Juan Francisco Berbeo. Bogotá: Universidad Pedagógica Nacional, LEBECS, tesis pregrado.

Grimson, A. (2011). Los límites de la cultura: crítica de las teorías de la identidad. Buenos Aires: Siglo XXI Editores.

Gutmann, A. (2008). La identidad en democracia. Madrid: Katz Editores.

Heredia, M. I. (2012). Saberes técnicos y saberes políticos sobre la educación en Colombia, 1978-1994. Bogotá: Pontificia Universidad Javeriana, tesis maestría en Historia.

Höffe, O. (2007). Ciudadano económico, ciudadano del Estado, ciudadano del mundo: ética política en la era de la globalización. Buenos Aires: Katz Editores.

Jiménez, D. A. (2011). Afrocolombianidad y educación: genealogía de un discurso educativo. Pedagogía y Saberes. 34, 87-103. Universidad Pedagógica Nacional.

Jiménez, D. A. (2006). Dinámicas y ejercicios de visibilización afrocolombianos en colegios distritales. Una aproximación. Bogotá: Universidad Nacional de Colombia, tesis de grado.

Línea Educación en Valores. (2008). Diseño de línea. Bogotá: Mimeo, Documento de Trabajo UPN.

Mejía, J., y Almanza, M. (2010). Comunidad LGBT: historia y reconocimientos jurídicos. Citas Latinoamericanas en Ciencias Sociales y Humanidades. Recuperado de http://132.248.9.1:8991/hevila/JusticiaBarraquilla/2010/no17/7.pdf

MEN. (2001). Cátedra de estudios afrocolombianos. Serie, lineamientos curriculares. Bogotá: Ministerio de Educación Nacional.

Nussbaum, M. (2010). Sin fines de lucro. Por qué la democracia necesita de las humanidades. Madrid: Katz Editores.

Pérez, T. (2014). Colombia de la educación en emergencia hacia una educación para el posconflicto y la paz, Rev.Interamericana de Investigación, educación y Pedagogía,Vol 7 №2 pág 287-311

Presidencia de la República. (2008). Décimo cuarto informe periódico presentado por el Estado colombiano para el Comité de la Naciones Unidas para la eliminación de todas la formas de violencia. Bogotá: Presidencia de la República. 
Rico, C. (2010). Apropiación ancestral y ambiental de la cuenca del Río Tunjuelito: una apuesta de formación ciudadana desde la prestación del servicio social escolar. Bogotá: Universidad Pedagógica Nacional, LEBESC, tesis pregrado.

Ricoeur, P. (2003). Lo justo. España: Caparrós Editores.

Santos, B. D. (1998). De la mano de Alicia. Lo social y lo político en la posmodernidad. Bogotá: Siglo del Hombre Editores y Universidad de los Andes.

Santos, B. D. (2005). Reinventar la Democracia. Reinventar el Estado. Buenos Aires: Clacso.

Sartori, G. (1994). ¿Qué es la democracia? Bogotá: Altamir Ediciones.

Sen, A. (2006). Desarrollo y libertad. Bogotá: Planeta.

Sierra, D. (2010). Empoderamiento de los derechos humanos, secuales y reproductivos: una herramienta para el ejercicio de la ciudadanía. Bogotá: Universidad Pedagógica Nacional, LEBECS, tesis pregrado.

Touraine, A. (1997). ¿Podremos vivir juntos? México: Fondo de Cultura Económica.

Ulloa, A. (2001). El nativo ecológico: movimientos indígenas y medio ambiente en Colombia. Recuperado de http://www.antropologia.com.br/arti/colab/a26-aulloa.pdf

Williams, R. (2003). Palabras clave. Un vocabulario de la cultura y la sociedad. Buenos Aires: Nueva Visión. 